Adv. Theor. Math. Phys. 2 (1998) $1441-1462$

\title{
Kähler Cone Substructure
}

\author{
Eric Sharpe \\ Department of Physics \\ Box 90305 \\ Duke University \\ Durham, NC 27708 \\ ersharpe@cgtp.duke.edu
}

\begin{abstract}
To define a consistent perturbative geometric heterotic compactification the bundle is required to satisfy a subtle constraint known as "stability," which depends upon the Kähler form. This dependence upon the Kähler form is highly nontrivial - the Kähler cone splits into subcones, with a distinct moduli space of bundles in each subcone and has long been overlooked by physicists. In this article we describe this behavior and its physical manifestation.
\end{abstract}

\section{Introduction}

To specify a perturbative heterotic compactification, one must specify a bundle (or, more generally, a torsion-free sheaf) on the compact space. One cannot specify any bundle; rather, it must satisfy certain consistency conditions in order to get a supersymmetric low-energy theory.

One of the consistency conditions is that the bundle must satisfy an equation known as the Donaldson-Uhlenbeck-Yau equation. This equation depends nontrivially upon the metric. This metric dependence has long been ignored among string theorists, but is in fact nontrivial and quite important.

e-print archive: http://xxx.lanl.gov/abs/hep-th/9810064 
In this paper we examine the metric dependence of this consistency constraint on bundles in perturbative heterotic compactifications. In particular, on the Calabi-Yau manifolds appearing, the Kähler cone splits into subcones (or "chambers"), with a distinct moduli space of bundles associated to each subcone.

We begin in sections 2 and 3 by reviewing general constraints on geometric heterotic compactifications, and Mumford-Takemoto stability in particular. In section 4 we then review the relevant mathematical results concerning how the Kähler cone splits into subcones. Then in section 5 we examine the physical behavior that reproduces this mathematics. (Essentially, one gets a perturbative enhanced $U(1)$ gauge symmetry on subcone walls, and the change in the moduli space is realized by examining $D$ terms.) In section 6 we briefly consider the implications of these results for string duality. (For example, this behavior in heterotic K3 compactifications corresponds to previously unknown behavior of hyper plets in type IIA Calabi-Yau compactifications at intermediate type IIA string coupling.) Finally in section 7 we work out a description of Kähler cone substructure on K3s that depends only on the Riemannian metric, not the precise complex structure, and also conjecture how this phenomenon generalizes when the Kähler cone is complexified by adding a $\mathrm{B}$ field. We also include a few appendices containing general background on moduli space problems and certain technical derivations.

Although in principle similar remarks hold in general, in this paper we will only consider bundles on surfaces (and typically only on K3s), not in other dimensions. We shall also only consider $G L(n, \mathbf{C})$ bundles, not bundles with more general structure groups. Finally, for most of this paper we shall only explicitly refer to the classical Kähler cone, not the complexified Kähler cone. Only towards the end will we explicitly study the effect of adding a B field.

In this article, when we speak of stability we shall always be referring to Mumford-Takemoto stability.

\section{Rapid review of heterotic compactifications}

For a consistent perturbative compactification of either the $E_{8} \times E_{8}$ or $\operatorname{Spin}(32) / \mathbf{Z}_{2}$ heterotic strings, in addition to specifying a Calabi-Yau $Z$ one must also specify a set of holomorphic vector bundles (or, more generally, 
torsion-free sheaves $\left.{ }^{1}\right) V_{i}$. These vector bundles must obey two constraints. For $G L(n, \mathbf{C})$ bundles one constraint can be written as

$$
J^{n-1} \cdot c_{1}\left(V_{i}\right)=0
$$

where $n$ is the complex dimension of the Calabi-Yau and $J$ is the Kähler form. Put another way, given a set of set of connections associated to local coordinate trivializations that are Hermitian (meaning, $F_{i j}=F_{\bar{\imath}}=0$ ), the constraint can be written as

$$
g^{i \bar{\jmath}} F_{i \bar{\jmath}}=0
$$

or, equivalently,

$$
F \wedge J^{n-1}=0
$$

This is known as the Donaldson-Uhlenbeck-Yau equation [31, section 15.6.2].

This constraint has a somewhat subtle implication. In general for any holomorphic bundle $\mathcal{E}$, if there exists a Hermitian connection associated to $\mathcal{E}$ such that, in every coordinate chart, the curvature $F$ satisfies $F \wedge$ $J^{n-1}=c I$, where $I$ is the identity matrix and $c \in \mathbf{R}$ is a fixed chartindependent constant, then $\mathcal{E}$ is either properly Mumford-Takemoto stable ${ }^{2}$, or Mumford-Takemoto semistable and split $[4,5,6]$. Thus, the constraint in equation (1) implies that (but is not equivalent to the statement) $\mathcal{E}$ is either stable, or semistable and split. In fact we can slightly simplify this statement. Properly semistable sheaves are grouped ${ }^{3}$ in $S$-equivalence classes, and each $S$-equivalence class contains a unique split representative [2, p. 23].

Thus, constraint (1) implies that $\mathcal{E}$ is Mumford-Takemoto semistable. Moreover, the constraint implies that the representative of any $S$-equivalence class that is relevant for physics is the unique split representative.

The other constraint is an anomaly-cancellation condition which, if a single $G L(r, \mathbf{C})$ bundle $V_{i}$ is embedded in each $E_{8}$, is often written as

$$
\sum_{i}\left(c_{2}\left(V_{i}\right)-\frac{1}{2} c_{1}\left(V_{i}\right)^{2}\right)=c_{2}(T Z)
$$

\footnotetext{
${ }^{1}$ We mention sheaves for completeness, though to aid readability in this paper we will only refer to bundles.

${ }^{2}$ We shall explain stability momentarily.

${ }^{3}$ More precisely, points on a moduli space of sheaves that are properly semistable do not necessarily correspond to unique semistable sheaves, but rather to " $S$-equivalence classes" of properly semistable sheaves. Points that are stable do correspond to unique stable sheaves - $S$-equivalence classes are a phenomenon arising only for properly semistable objects. For more information, see appendix A.
} 
It was noted [9] that the anomaly-cancellation conditions can be modified slightly by the presence of five-branes in the heterotic compactification. However, we shall only be concerned with perturbative heterotic compactifications in this paper.

\section{Mumford-Takemoto stability}

In the previous section we mentioned that for a consistent perturbative heterotic compactification, the holomorphic bundle on the Calabi-Yau must be "Mumford-Takemoto semistable." What does this mean?

For any torsion-free sheaf $\mathcal{E}$, define the slope of $\mathcal{E}$ to be

$$
\mu(\mathcal{E})=\frac{c_{1}(\mathcal{E}) \cdot J^{n-1}}{\operatorname{rank} \mathcal{E}}
$$

where $J$ is the Kähler form. We will sometimes use the notation $\mu_{J}(\mathcal{E})$ when there is ambiguity in the choice of Kähler form $J$. We say $\mathcal{E}$ is MumfordTakemoto (semi)stable if, for all proper coherent subsheaves $\mathcal{F} \subset \mathcal{E}$ such that $0<\operatorname{rank} \mathcal{F}<\operatorname{rank} \mathcal{E}$ and $\mathcal{E} / \mathcal{F}$ is torsion-free, we have

$$
\mu(\mathcal{F})(\leq)<\mu(\mathcal{E})
$$

Note that if $\mathcal{E}$ is a torsion-free sheaf such that $c_{1}(\mathcal{E})=0$ and if $\mathcal{E}$ has sections, then it can be at best semistable, not strictly stable. This is because the section defines a map $\mathcal{O} \rightarrow \mathcal{E}$, so we have a subsheaf $\mathcal{F}$ (namely, $\mathcal{F}=\mathcal{O}$ ) such that $\mu(\mathcal{F})=0=\mu(\mathcal{E})$.

In passing, we should make a technical remark concerning torsion-free sheaves that are not bundles. The stability constraint was originally derived from the low-energy supergravity for geometric compactifications involving bundles, not more general sheaves. Although one can certainly define Mumford-Takemoto (semi)stability for other sheaves (as we have done, in fact), there are other, inequivalent notions of stability (prominently, Gieseker stability), and it is not clear whether Mumford-Takemoto stability is the correct notion of stability for heterotic compactifications involving sheaves that are not bundles. For nongeometric compactifications, even less is known no one knows any analogue of the stability constraint. 


\section{Kähler cone substructure}

Note that Mumford-Takemoto stability depends implicitly upon the choice of Kähler form [2, 3, 11, 12, 13,14,15,16,17, 18,19,20]. This choice is extremely important - sheaves that are stable with respect to one Kähler form may not be stable with respect to another. In general, for fixed Chern classes a moduli space of sheaves will not have the same form everywhere inside the Kähler cone, but rather will have walls along which extra sheaves become semistable. These walls stratify the Kähler cone into subcones (or "chambers"), inside any one of which the notion of stability is constant.

We should take a moment to clarify these remarks slightly. In typical circumstances, a generic stable bundle will be stable for all choices of Kähler form. However, the stability of some nongeneric subset will depend nontrivially upon the Kähler form, and so the moduli space will change as one wanders around in the Kähler cone. More precisely, as the Kähler moduli are varied, some (typically nongeneric) stable bundles will become strictly semistable, then unstable, and vice-versa.

In this section we shall describe, without proof, necessary (but not sufficient ${ }^{4}$ if the rank is greater than 2) conditions for chamber walls inside the Kähler cone. (For the special case of moduli spaces of rank 2 sheaves, it is known that these conditions are both necessary and sufficient for the moduli space to change.)

Define the discriminant of a coherent sheaf $\mathcal{E}$ on an algebraic Kähler surface to be

$$
\Delta(\mathcal{E})=2 r c_{2}(\mathcal{E})-(r-1) c_{1}(\mathcal{E})^{2}
$$

where $r$ is the rank of $\mathcal{E}$. It can be shown (see $[2$, section 3.4] or $[3,30]$ ) that when $\mathcal{E}$ is Mumford-Takemoto semistable, $\Delta(\mathcal{E}) \geq 0$.

Walls inside the Kähler cone $K$ are specified by divisors $\zeta$ satisfying certain conditions. For a given divisor $\zeta$, the corresponding wall is

$$
W_{\zeta}=\{J \in K \mid \zeta \cdot J=0\}
$$

(Note that by the Hodge index theorem, on an algebraic Kähler surface the positive definite part of $H^{1,1}$ is one-dimensional, so the intersection form on $H^{1,1}$ has signature $(+,-, \cdots,-)$.)

\footnotetext{
${ }^{4}$ More precisely, the conditions we shall state are necessary (but in general not sufficient) for the moduli space to change. For rank greater than two, sufficient conditions on chamber walls for the moduli space to change are not known.
} 
Precisely which divisors $\zeta$ can define chamber walls? The conditions $[19,20]$ are that, for some integer $i, 0<i<r$,

$$
\begin{aligned}
& \text { 1) } \zeta=r F-i c_{1} \text { for some divisor } F \\
& \text { 2) }-i(r-i) \Delta \leq \zeta^{2}<0
\end{aligned}
$$

We shall not attempt to completely prove this result here (but for a detailed examination, see appendix B). However, part of this result is relatively clear. Suppose $\mathcal{E}$ is a rank $r$ bundle that is stable in some parts of the Kähler cone and unstable in others. Let $\mathcal{F}$ be a potentially destabilizing subsheaf of $\mathcal{E}$, in the sense that when $\mathcal{E}$ becomes unstable as a function of the Kähler form $J, \mu_{J}(\mathcal{F})$ grows to become larger than $\mu_{J}(\mathcal{E})$. Define $F=c_{1}(\mathcal{F}), i=\operatorname{rank} \mathcal{F}$. Then the condition on $J$ for the bundle $\mathcal{E}$ to be strictly semistable is $\mu_{J}(\mathcal{F})=\mu_{J}(\mathcal{E})$, which we can rewrite as $J \cdot\left(r F-i c_{1}\right)=0$. Put another way, if $\mathcal{E}$ is strictly semistable for some Kähler form $J$ then there exists a divisor $F$ and an integer $i$, such that $\zeta=r F-i c_{1}$ and $J \cdot \zeta=0$.

Some examples might help the reader. Consider a generic elliptic K3 with section. Its Kähler cone has two generators, corresponding to the section $S$ and the fiber $F$, obeying $S^{2}=-2, F^{2}=0$, and $S \cdot F=1$. Write $J=a S+b F$, then the Kähler cone is defined by the inequalities $a>0$ and $b>2 a$. Consider a moduli space of rank 2 bundles of $c_{1}=0$ and $c_{2}=4$. In this case it is straightforward to show that the Kähler cone splits into two subcones, with the chamber wall located along Kähler forms proportional to $S+3 F$. For another example, consider a moduli space consisting of rank 2 bundles of $c_{1}=0$ and $c_{2}=24$ (the moduli space containing the tangent bundle), on the same K3. It is straightforward to check that the Kähler cone splits into 15 subcones in this case.

In certain cases it is possible to see Kähler cone substructure explicitly. For example, in moduli spaces of equivariant sheaves on toric varieties, this substructure is essentially manifest. We shall not work through such examples in this paper; see instead [25, 26].

Moduli spaces associated to distinct chambers of a Kähler cone are often, but not always, birational to each other. (If they are not birational, then it is because at least one is reducible, and in crossing the wall an entire component either appeared or disappeared.) We shall see explicitly how distinct moduli spaces are related in the next section.

In passing, we should note that the behavior of Mumford-Takemoto stability as the Kähler form changes is closely related to the behavior of GIT quotients under change of polarization [21, 22]. 
We should also point out that this behavior is closely related to the behavior of Donaldson polynomial invariants on manifolds of $b_{2}^{+}=1$ as the metric changes. There also, one finds walls in the space of metrics. (However, in Donaldson theory this only happens on four-manifolds of $b_{2}^{+}=1$, whereas the Kähler cone substructure described in this paper potentially occurs on any algebraic variety.) In the present discussion we fix Chern classes and examine the behavior of moduli spaces of bundles of those fixed Chern classes as the Kähler form changes, whereas in Donaldson theory one sums over contributions from different Chern classes. In essence, Donaldson theory is a "topological" version of the "algebro-geometric" phenomenon being discussed here. For more information on Donaldson theory on manifolds of $b_{2}^{+}=1$, see for example $[23,24]$.

\section{Physics at chamber walls}

Suppose we have a heterotic compactification involving some bundle $\mathcal{E}$, and we have varied the Kähler form until the bundle $\mathcal{E}$ is no longer stable ${ }^{5}$, but rather properly semistable. What does this mean for the low-energy theory? The low-energy theory picks up an enhanced $U(1)$ gauge symmetry. The transformation of the moduli space of bundles is encoded in D terms.

Why does the low-energy effective theory get an enhanced $U(1)$ gauge symmetry? Properly semistable bundles occur on a moduli space in $S$ equivalence classes (see appendix A), and each $S$-equivalence class contains a unique split representative [2, p. 23]. Moreover, as discussed earlier, the representative of the $S$-equivalence class relevant for physics is the split representative. Thus, if $\mathcal{E}$ is a properly semistable bundle, then the physically relevant representative of the same $S$-equivalence class can be written in the form $\mathcal{E}=\mathcal{F} \oplus \mathcal{G}$ for semistable $\mathcal{F}, \mathcal{G}$. Since the bundle splits, we have a perturbative enhanced $U(1)$ gauge symmetry. This is because the lowenergy gauge theory is the largest subgroup of the ten-dimensional gauge group that commutes with the structure group of the bundle. When the bundle splits, its structure group can be reduced from $S U(n)$ (where $n$ is its rank) to $S\left[U\left(n_{1}\right) \times U\left(n_{2}\right)\right]$, where $n=n_{1}+n_{2}$. Everything that commuted with $S U(n)$ also commutes with $S\left[U\left(n_{1}\right) \times U\left(n_{2}\right)\right]$, and in addition there is an extra commuting $U(1)$ factor, described explicitly by $S U(n)$ matrices of the form $\operatorname{diag}(x, x, \cdots, x, y, y, \cdots y)$, with $x^{n_{1}} y^{n_{2}}=1$. Thus, there is a perturbative enhanced $U(1)$ gauge symmetry ${ }^{6}$ in the low-energy effective

\footnotetext{
${ }^{5}$ In typical cases, this can only happen for certain nongeneric $\mathcal{E}$.

${ }^{6}$ In fact, we are being slightly sloppy - one sometimes will also need to mod out by finite groups - but this will not affect our analysis. It is relatively straightforward to see
} 
theory.

Since the low-energy theory has picked up a $U(1)$, we now have to worry about $\mathrm{D}$ terms, and indeed these will explicitly realize the moduli space behavior mentioned earlier. In order to see this behavior explicitly, let us examine the chiral fields of the low-energy theory which will be charged under the $U(1)$.

Write $\mathcal{E}=\mathcal{F} \oplus \mathcal{G}$, then deformations of $\mathcal{E}$ (classified by elements of ${ }^{7}$ $\left.H^{1}(\operatorname{End} \mathcal{E})=H^{1}(\operatorname{Hom}(\mathcal{E}, \mathcal{E}))\right)$ have contributions from four sources:

$$
\begin{aligned}
& H^{1}(\operatorname{Hom}(\mathcal{F}, \mathcal{F})) \\
& H^{1}(\operatorname{Hom}(\mathcal{G}, \mathcal{G})) \\
& H^{1}(\operatorname{Hom}(\mathcal{F}, \mathcal{G})) \\
& H^{1}(\operatorname{Hom}(\mathcal{G}, \mathcal{F}))
\end{aligned}
$$

The first two contributions - namely, deformations of $\mathcal{F}$ and $\mathcal{G}$ individually - are neutral under the $U(1)$. The second pair of contributions, which mix $\mathcal{F}$ into $\mathcal{G}$ and vice-versa - have equal and opposite charges under the $U(1)$.

In order to make notation more concise, let us define

$$
\begin{aligned}
\alpha_{i} & \in H^{1}(\operatorname{Hom}(\mathcal{F}, \mathcal{G})) \\
\beta_{j} & \in H^{1}(\operatorname{Hom}(\mathcal{G}, \mathcal{F}))
\end{aligned}
$$

and identify $\alpha_{i}, \beta_{j}$ with the corresponding chiral superfields.

We can now write the $\mathrm{D}$ term ${ }^{8}$ of the low-energy effective action associ-

these factors. Suppose that we have embedded $S U(3)$ in an $E_{8}$. The group $E_{8}$ contains a subgroup [10]

$$
\frac{E_{6} \times S U(3)}{\mathbf{Z}_{3}}
$$

and so this is the reason why the largest group commuting with $S U(3)$ is $E_{6}$ rather than $E_{6} \times Z(S U(3))\left(Z(S U(3))=\mathbf{Z}_{3}\right)$ - the center of $S U(3)$ is identified with a $\mathbf{Z}_{3}$ subgroup of $E_{6}$. If $S U(3)$ is reduced to $S[U(2) \times U(1)]$, then the low-energy gauge group is actually

$$
\frac{E_{6} \times U(1)}{\mathbf{Z}_{3}}
$$

We would like to thank A. Knutson for an explanation of this detail.

${ }^{7}$ Infinitesimal deformations of an arbitrary torsion-free sheaf $\mathcal{E}$ are classified by elements of $\operatorname{Ext}^{1}(\mathcal{E}, \mathcal{E})[7,8]$, however for the purposes of making this paper more readable, we usually restrict to bundles, and for $\mathcal{E}$ a bundle, $\operatorname{Ext}^{1}(\mathcal{E}, \mathcal{E})=H^{1}($ End $\mathcal{E})$.

${ }^{8}$ In this paper we concentrate on K3 compactifications, and so we will get a triplet of $\mathrm{D}$ terms, not a single $\mathrm{D}$ term. However, to simplify the presentation, we shall momentarily forget this point and only consider a single $\mathrm{D}$ term. 
ated to the enhanced $U(1)$ in the form

$$
D=\sum_{i=1}^{n}\left|\alpha_{i}\right|^{2}-\sum_{j=1}^{m}\left|\beta_{j}\right|^{2}-r
$$

where $r$ is some function of the Kähler moduli, and

$$
\begin{aligned}
n & =\operatorname{dim} H^{1}(\operatorname{Hom}(\mathcal{F}, \mathcal{G})) \\
m & =\operatorname{dim} H^{1}(\operatorname{Hom}(\mathcal{G}, \mathcal{F}))
\end{aligned}
$$

The analysis of these $\mathrm{D}$ terms is now essentially identical to that presented in [27]. Depending upon the values of $n$ and $m$, there are essentially three distinct cases to consider:

1) $n=m=0$

This trivial case occurs when locally all deformations of the bundle preserve its splitness. We shall not speak to this case further.

2) $n>0, m=0$

In this case, when $r>0$ the moduli space is locally $\mathbf{P}^{n}$, yet when $r<0$ the moduli space is empty. This is precisely the description of a component of a reducible moduli space appearing or disappearing at a chamber wall.

In the special case of bundles on K3s, however, $n=m$ by Serre duality, so case (2) cannot arise. (For a discussion of irreducibility of moduli spaces of bundles on $\mathrm{K} 3 \mathrm{~s}$, a sufficient but not necessary condition for case (2) not to arise, see $[34,35]$.)

3) $n>0, m>0$

In this case, by varying $r$ we recover a birational transformation.

Recall from the previous section that moduli spaces of bundles associated to distinct chambers are either related by a birational transformation, or one is reducible and an entire component appears or disappears at a chamber wall. We have explicitly recovered these possibilities ${ }^{9}$ in terms of D terms associated to the enhanced $U(1)$.

Now let us specialize to the case of a heterotic K3 compactification. In this case in the low energy supergravity the moduli are in hyperplets, and $\mathrm{D}$ terms come in triplets. How does the above story specialize? First, recall that a charged hyperplet can be thought of as a pair of oppositely charged

\footnotetext{
${ }^{9}$ We would like to thank E. Witten for a useful discussion of this matter.
} 
chiral plets. In the present case, using Serre duality we have

$$
H^{1}(\operatorname{Hom}(\mathcal{F}, \mathcal{G})) \cong H^{1}(\operatorname{Hom}(\mathcal{G}, \mathcal{F}))^{\vee}
$$

so clearly the moduli that become charged under the $U(1)$ at the subcone boundary fill out hyperplets.

We should clarify a few additional points. A deformation by an element of $H^{1}(\operatorname{Hom}(\mathcal{F}, \mathcal{G}))$ or $H^{1}(\operatorname{Hom}(\mathcal{G}, \mathcal{F}))$ will define a distinct bundle only up to an overall scale factor (see appendix $\mathrm{C}$ ), so we get a total of 2 complex bosons (one hyperplet) more than we would have gotten from $H^{1}(E n d \mathcal{E})$ for generic $\mathcal{E}$. Indeed, one expects ${ }^{10}$ light vectors to be paired with light hyper plets, and as we have an enhanced $U(1)$, we should not be surprised to find an extra hyper plet.

In addition, matter charged under the rest of the low-energy gauge group can also become charged under the $U(1)$. Recall from [37] that $H^{1}(\mathcal{E})$ counts complex bosons charged under part of the (generic) low-energy gauge group (phrased differently, $(1 / 2) h^{1}(\mathcal{E})$ is the number of charged hyper plets). In the case that $\mathcal{E}=\mathcal{F} \oplus \mathcal{G}$, these will split into $h^{1}(\mathcal{F})$ charged complex scalars and $h^{1}(\mathcal{G})$ charged complex scalars, each charged oppositely under the enhanced $U(1)$.

In passing, we should also point out that in heterotic compactifications on $\mathrm{K} 3, \mathrm{D}$ terms come in triplets, a fact we have essentially ignored so far in this section. One implication of this fact is that Kähler cone substructure on K3s can be understood in a complex-structure-invariant fashion; this will be discussed in section 7 .

We have also glossed over anomaly cancellation so far in this section. In fact, the enhanced $U(1)$ appearing at chamber walls is anomalous, and gets a mass through a six-dimensional version of the Dine-Seiberg-Witten mechanism [32], as explained in [33]. The three real scalars forming the triplet of Fayet-Iliopoulos terms in our discussion above form three-fourths of a hyper plet. The fourth real scalar of this hyper plet gains a translation symmetry, gauged by the $U(1)$, an artifact of Green-Schwarz anomaly cancellation in ten dimensions. (This fourth scalar descends from the two-form tensor field in ten dimensions, and its translation symmetry is a relic of the anomalous transformation of the ten-dimensional tensor as assigned in the Green-Schwarz mechanism.) Because of this gauged translation symmetry,

\footnotetext{
${ }^{10}$ If the $U(1)$ were nonanomalous, then this would follow immediately from anomaly factorization - if $n_{H}$ is the number of hyper plets and $n_{V}$ the number of vectors, then one needs $n_{H}-n_{V}=244$ [36]. However, we shall see shortly that the $U(1)$ receives a mass through the Dine-Seiberg-Witten mechanism, so an anomaly factorization argument is not really appropriate.
} 
the $U(1)$ vector field is actually massive. In particular, when checking sixdimensional Green-Schwarz anomaly factorization, the $U(1)$ should not be counted.

\section{$6 \quad$ String duality}

What can we learn by applying string duality to Kähler cone substructure?

First, the attentive reader might be very concerned about F-theory duals to heterotic K3 compactifications. The Kähler cone substructure described in this paper has not been seen in F-theory to date; why not?

Suppose we have compactified the heterotic theory on an elliptic K3 with section. Then, loosely speaking, it turns out [11] that there is a unique chamber corresponding to the case that the section is much larger than the fiber. This particular chamber is the one that has been sensed by F-theory arguments to date.

For example, in the recent past a description of bundles on elliptic CalabiYaus was worked out by Friedman, Morgan, Witten [38]. They explicitly assume that the Kähler form lies in the distinguished chamber described above, namely, that the elliptic fiber is much smaller than all other Kähler moduli. (Having made this assumption in the beginning, the rest of their paper is written without any reference to Kähler forms.)

Precisely what does the chamber structure dualize to? For technical simplicity, consider a heterotic theory compactified on $K 3 \times T^{2}$, dual to type IIA on some Calabi-Yau threefold. Assume for simplicity that the $\mathrm{K} 3$ is elliptic with section. Then the weakly coupled IIA string is dual to a heterotic theory with a big section [39]. Put another way, the weakly coupled IIA string is dual to the heterotic theory in the distinguished chamber described above. As we move to the boundary of the chamber, the IIA string stops being weakly coupled. Thus, it appears (somewhat loosely) that the chamber structure of the perturbative heterotic string dualizes to new behavior in the hyperplet moduli space at intermediate IIA string coupling.

Since the IIA dual to Kähler cone substructure occurs at intermediate string coupling, one probably cannot hope to find a geometric explanation in terms of the IIA Calabi-Yau. However, if one considered M theory compactifications on a Calabi-Yau threefold, dual to the heterotic string on $K 3 \times S^{1}$, then it might be possible to see heterotic Kähler cone substructure in terms of the geometry of the Calabi-Yau. We shall not pursue this direction here, 
however.

\section{Complex structure ambiguity on K3s}

In most of this paper we have referred to bundles that are holomorphic with respect to a specific complex structure. On K3s we should really be slightly more careful. As K3s are hyperKähler manifolds, they possess Ricciflat metrics that are Hermitian with respect to multiple complex structures. The choice of complex structure is not physically meaningful, and in heterotic compactifications on $\mathrm{K} 3$ this ambiguity is reflected in the $S U(2) \mathrm{R}$-symmetry of the six dimensional supergravity.

As the low-energy supergravity possesses an $S U(2)$ R-symmetry that reflects a physical invariance under rotation of complex structure, one expects that there should exist a complex-structure-invariant description of Kähler cone substructure. More precisely, there should exist a description of Kähler cone substructure that refers only to the metric and Chern classes, not to any particular complex structure.

There is another reason to believe that there should exist a complexstructure-invariant formulation of Kähler cone substructure on K3s. By following the arguments of [31, section 15.6], one finds that the metric and connection must satisfy the Donaldson-Uhlenbeck-Yau equation (2) for not just one complex structure, but for each complex structure on the K3. Put another way, one has a triplet of constraints of the form

$$
F \wedge J^{i j}=0
$$

where $J^{i j}$ is a triplet of Kähler forms, transforming under the symmetric representation of $S U(2)_{R}$.

Such a complex-structure-invariant description does exist, and we shall describe it here, following the notation and conventions of [28, 29].

First, we shall consider how Kähler cone substructure is specified on the space of Ricci-flat metrics on K3, namely the Grassmannian of spacelike 3planes in $\mathbf{R}^{3,19}$. Suppose we are studying moduli spaces of bundles of rank $r$ and Chern classes $c_{1}, c_{2}$. We shall make the (simplifying) assumption that $c_{1}=0$. (Note that if $c_{1}$ were nonzero, then it would implicitly fix a complex structure, namely that in which $c_{1} \in H^{2}(K 3, \mathbf{Z}) \cap H^{1,1}(K 3, \mathbf{R})$.) Let $\zeta \in \Gamma^{3,19}$ such that for some integer $i, 0<i<r$,

$$
-i(r-i)\left(2 r c_{2}\right) \leq r^{2} \zeta^{2}<0
$$


then in the space of Riemannian metrics (defined by spacelike 3-planes $\Sigma \subset$ $\Gamma^{3,19}$ ) there is a wall defined by $\zeta$, as

$$
\left.W_{\zeta}=\left\{\Sigma \in \text { Grassmannian(spacelike 3-planes in } \mathbf{R}^{3,19}\right) \mid \Sigma \cdot \zeta=0\right\}
$$

and along this wall, for certain nongeneric bundles, one will get enhanced $U(1)$ gauge symmetries in the low-energy theory.

Specific complex structures are then assigned to a Riemannian metric $\Sigma$ by picking a specific spacelike 2-plane $\Omega \subset \Sigma$, and the Kähler form $J$, modulo rescalings, is simply the orthogonal complement of $\Omega$ in $\Sigma$. For any given choice of $\Omega$, this description correctly reproduces the Kähler cone substructure described earlier, and so this is clearly the desired complexstructure-invariant formulation.

If one is somewhat braver, one can make a natural conjecture concerning how this situation changes when one adds a $B$ field. In this case, let $\zeta \in \Gamma^{4,20}$ be such that, for some integer $i, 0<i<r$,

$$
-i(r-i)\left(2 r c_{2}\right) \leq r^{2} \zeta^{2}<0
$$

Metrics and $B$ fields are combined into a spacelike 4-plane $\Pi \subset \mathbf{R}^{4,20}$. It then seems quite reasonable that walls in the space of spacelike 4-planes are defined by

$$
\left.W_{\zeta}=\left\{\Pi \in \text { Grassmannian(spacelike 4-planes in } \mathbf{R}^{4,20}\right) \mid \Pi \cdot \zeta=0\right\}
$$

How do we then break down $\Pi$ into a specific choice of metric and $B$ field ? Pick $w \in \Gamma^{4,20}$ such that $w^{2}=0$ and $w \cdot \zeta=0$. Define $\Sigma^{\prime}=\Pi \cap w^{\perp}$, then a metric is defined by a spacelike 3-plane $\Sigma$ which is simply the image of $\Sigma^{\prime}$ in $w^{\perp} / w$. In order to find a corresponding value of $B$, pick $w^{*} \in \Gamma^{4,20}$ such that $\left(w^{*}\right)^{2}=0, w \cdot w^{*}=1, w^{*} \perp w^{\perp}$, and $w^{*} \cdot \zeta=0$. Let $B^{\prime} \in \mathbf{R}^{4,20}$ such that $B^{\prime} \cdot \Sigma^{\prime}=0$ and $B^{\prime} \cdot w=1$, then project $B^{\prime}$ into $w^{\perp} / w$ to get $B$.

Note that this condition on the conformal field theory is very closely related to the condition for an enhanced nonabelian gauge symmetry in type IIA compactifications on K3 [29].

\section{Conclusions}

In this paper we have studied the "stability" of bundles, a necessary condition for a consistent perturbative heterotic compactification, and in particular examined the dependence of stability upon the Kähler form. The Kähler 
cone splits into subcones, which is reflected physically in the appearance of an enhanced $U(1)$ gauge symmetry at a subcone wall (for a compactification involving a (nongeneric) bundle whose stability changes at the wall). In particular, we have outlined known mathematical results concerning the positions of these walls, and also examined how their appearance is reflected physically.

There are several directions that remain to be pursued. For example, we have not studied Kähler cone substructure on Calabi-Yaus of dimension greater than two. To our knowledge, mathematical results on stability for algebraic varieties of dimension greater than two are extremely limited (though it may be possible to empirically derive some conjectures by using equivariant sheaves $[25,26]$ on toric varieties, for which Kähler cone substructure appears manifestly.)

We also have not studied bundles with structure group other than $G L(n, \mathbf{C})$. Presumably related phenomena appear in spaces of bundles of other structure groups; it would be interesting to determine precisely what phenomena occur.

\section{Acknowledgements}

We would like to thank P. Aspinwall, R. Friedman, T. Gomez, A. Knutson, G. Moore, J. Morgan, D. Morrison, R. Plesser, Z. Qin, and E. Witten for useful discussions.

\section{A Notes on moduli spaces of bundles}

In order to get a well-behaved moduli space, one needs some notion of "stability." For example, consider the analogous (and much simpler) problem of constructing $\mathbf{P}^{1}$ as a quotient of $\mathbf{C}^{2}$ by $\mathbf{C}^{\times}$:

$$
\mathbf{P}^{1}=\frac{\mathbf{C}^{2}-\{0\}}{\mathbf{C}^{\times}}
$$

In order to get a well-behaved result, one first removes the point 0 from $\mathbf{C}^{2}$ before quotienting by $\mathbf{C}^{\times}$. Technically this construction of $\mathbf{P}^{1}$ is known as a Geometric Invariant Theory (GIT) quotient, and the point $0 \in \mathbf{C}^{2}$ is an example of an unstable point. 
Alternatively, one can construct $\mathbf{P}^{1}$ as

$$
\mathbf{P}^{1}=\frac{S^{3}}{U(1)}
$$

In this construction, we do not need to remove a point before quotienting; rather, we first restrict to a cross-section of $\mathbf{C}^{2}$, and then quotient by a smaller group. This construction is known formally as a symplectic quotient, and as is well-known, GIT quotients give the same results as symplectic quotients. In symplectic quotients one does not define a notion of "stability" - one picks out a slice at the beginning that never intersects any "unstable" points.

Strictly speaking string theory uses a description of moduli spaces closely analogous to a symplectic quotient - solutions of the Donaldson-UhlenbeckYau equation. However, this analogue of a symplectic quotient has an equivalent formulation in terms of an analogue of a GIT quotient, and it is the GIT quotient formulation that most people refer to.

So far we have considered the gross features of moduli spaces, however for this paper we shall need a little more information. It is possible for a point to be stable, but only just barely - this is often referred to as being semistable. Semistable objects do not map one-to-one into the moduli space; rather, to get well-behaved results, several semistable objects are typically identified with a single point on the moduli space. In the context of moduli spaces of bundles, such classes of semistable holomorphic bundles are known as $S$ equivalence classes. (In the context of symplectic quotients, this corresponds to a symplectic reduction at a nonregular value of the moment map.)

Now let us see how $S$-equivalence classes arise a little more explicitly. Consider deforming $\mathcal{E}=\mathcal{F} \oplus \mathcal{G}$ by an element of $H^{1}(\operatorname{Hom}(\mathcal{F}, \mathcal{G}))$ only - do not turn on any elements of $H^{1}(\operatorname{Hom}(\mathcal{G}, \mathcal{F}))$. Then it turns out (see appendix $\mathrm{C}$ ) that the overall scale does not matter - elements of $H^{1}(\operatorname{Hom}(\mathcal{F}, \mathcal{G}))$ which differ only by a scale define isomorphic bundles.

Now, by scaling an element of $H^{1}(\operatorname{Hom}(\mathcal{F}, \mathcal{G}))$ down to zero, we recover a one-parameter family of bundles, of the form

$$
\mathcal{E}_{t}= \begin{cases}\mathcal{E}^{\prime} & t \neq 0 \\ \mathcal{F} \oplus \mathcal{G} & t=0\end{cases}
$$

If we define a moduli space of bundles in such a way that $\mathcal{E}^{\prime}$ and $\mathcal{E}_{0}=\mathcal{F} \oplus \mathcal{G}$ are distinct, then as $\mathcal{E}_{0}$ is the limit of a one-parameter family describing $\mathcal{E}^{\prime}$, the moduli space cannot be Hausdorff ${ }^{11}$. It is therefore much more natural

\footnotetext{
${ }^{11}$ Technical experts would no doubt prefer we use the term "separable" rather than
} 
to associate $\mathcal{E}^{\prime}$ and $\mathcal{E}_{0}$ with the same point on the moduli space, thereby getting a much better behaved moduli space. The pair consisting of $\mathcal{E}^{\prime}$ and $\mathcal{E}_{0}$ is an example of an $S$-equivalence class, and this example has hopefully demonstrated why one uses $S$-equivalence classes to define moduli spaces.

In the text, we have discussed how stability depends upon the Kähler form. What happens to $S$-equivalence classes as the Kähler form is varied? Let $\mathcal{E}$ be a bundle that is stable with respect to some Kähler forms and unstable with respect to others, so that along some wall in the Kähler cone it is strictly semistable. As we have just discussed, along that wall in the Kähler cone, $\mathcal{E}$ will lie in a nontrivial $S$-equivalence class of bundles. What typically happens is that the other elements of the $S$-equivalence class are strictly semistable only for Kähler forms along that wall, and are unstable for other Kähler forms. So, if the Kähler form is moved off the wall in such a way that $\mathcal{E}$ becomes stable, then the other elements of the $S$-equivalence class become unstable and so drop out of the picture, leaving one with a unique representative.

\section{B Derivation of chamber walls}

In this section we shall derive the necessity conditions for a chamber wall in the Kähler cone, in a special case. This derivation is already known (see for example [19]); however, as the methods are unfamiliar to most physicists, we repeat it here.

Before working through the derivation, we need a few standard results. Given a torsion-free coherent sheaf, there exists a filtration of $\mathcal{E}[2,3]$

$$
0=\mathcal{E}_{0} \subset \mathcal{E}_{1} \subset \cdots \subset \mathcal{E}_{n}=\mathcal{E}
$$

(where $\subset$ indicates proper subsheaf) known as the Harder-Narasimhan filtration, with the properties

(1) $\mathcal{E}_{i} / \mathcal{E}_{i-1}$ is semistable for $1 \leq i \leq n$

(2) $\mu\left(\mathcal{E}_{i} / \mathcal{E}_{i-1}\right)>\mu\left(\mathcal{E}_{i+1} / \mathcal{E}_{i}\right)$ for $1 \leq i \leq n-1$

For every torsion-free coherent sheaf $\mathcal{E}$, a Harder-Narasimhan filtration exists and, for fixed Kähler form, is unique.

Note that the Harder-Narasimhan filtration is trivial (meaning, of the form $0=\mathcal{E}_{0} \subset \mathcal{E}_{1}=\mathcal{E}$ ) precisely when $\mathcal{E}$ is semistable. Intuitively, the Harder-Narasimhan filtration gives information about the "instability" of $\mathcal{E}$.

"Hausdorff," however we have decided to forgo a small amount of technical accuracy in exchange for improved readability. 
Now, suppose $\mathcal{E}$ is a torsion-free coherent sheaf on an algebraic surface that is stable with respect to Kähler form $J_{1}$ and unstable with respect to Kähler form $J_{2}$. Suppose $\mathcal{E}$ has Harder-Narasimhan filtration (with respect to $J_{2}$ ) of the form

$$
0 \subset \mathcal{E}_{1} \subset \mathcal{E}
$$

This need not always be the case; however, we shall only study this case in this appendix. (The general case follows similarly $[19,20]$.) We have an exact sequence

$$
0 \rightarrow \mathcal{E}_{1} \rightarrow \mathcal{E} \rightarrow \mathcal{E} / \mathcal{E}_{1} \rightarrow 0
$$

Define the discriminant $\Delta(\mathcal{E})=2 r c_{2}(\mathcal{E})-(r-1) c_{1}(\mathcal{E})^{2}$, where $r=$ rank $\mathcal{E}$. By Bogomolov's inequality $[2,3,30]$, we have that $\Delta(\mathcal{E}) \geq 0$ if $\mathcal{E}$ is MumfordTakemoto semistable.

Define $F=c_{1}\left(\mathcal{E}_{1}\right), i=\operatorname{rank} \mathcal{E}_{1}$, and $\zeta=r F-i c_{1}(\mathcal{E})$. Use the identity

$$
\Delta(\mathcal{E})-\frac{r}{i} \Delta\left(\mathcal{E}_{1}\right)-\frac{r}{r-i} \Delta\left(\mathcal{E} / \mathcal{E}_{1}\right)=-\frac{\zeta^{2}}{i(r-i)}
$$

Now, as $\mathcal{E}_{1}$ and $\mathcal{E} / \mathcal{E}_{1}$ are $J_{2}$-semistable (from the definition of HarderNarasimhan filtration), we have that

$$
\Delta(\mathcal{E}) \geq-\frac{\zeta^{2}}{i(r-i)}
$$

We now merely need to show that $\zeta^{2}<0$. From the definition of HarderNarasimhan filtration, we have $\mu_{J_{2}}\left(\mathcal{E}_{1}\right)>\mu_{J_{2}}\left(\mathcal{E} / \mathcal{E}_{1}\right)$, which implies $J_{2} \cdot \zeta>$ 0 . As $\mathcal{E}$ is $J_{1}$-stable, we know that $\mu_{J_{1}}\left(\mathcal{E}_{1}\right)<\mu_{J_{1}}(\mathcal{E})$, which implies that $J_{1} \cdot \zeta<0$. Thus, there exists $J$ such that $J \cdot \zeta=0$, and so from the Hodge index theorem we have $\zeta^{2}<0$.

Thus, if $\mathcal{E}$ is $J_{1}$-stable but $J_{2}$-unstable, then there exists a divisor $\zeta$ such that, for some integer $i, 0<i<r$,

$$
\begin{aligned}
& \text { (1) } \zeta=r F-i c_{1}(\mathcal{E}) \text { for some divisor } F \\
& \text { (2) }-i(r-i) \Delta(\mathcal{E}) \leq \zeta^{2}<0
\end{aligned}
$$

and moreover $\mathcal{E}$ becomes strictly semistable for a Kähler form $J$ such that $J \cdot \zeta=0$. It should be clear that these conditions are necessary for a moduli space to change, but not necessarily sufficient. 


\section{Notes on $H^{1}(\operatorname{Hom}(\mathcal{F}, \mathcal{G}))$}

Suppose $\mathcal{F}$ and $\mathcal{G}$ are both bundles. Then we can define a bundle $\mathcal{E}$ as an extension of $\mathcal{F}$ by $\mathcal{G}$

$$
0 \rightarrow \mathcal{G} \rightarrow \mathcal{E} \rightarrow \mathcal{F} \rightarrow 0
$$

and extensions of this form are well-known to be classified by elements of $\operatorname{Ext}^{1}(\mathcal{F}, \mathcal{G})=H^{1}(\operatorname{Hom}(\mathcal{F}, \mathcal{G}))$

There is, however, a subtlety: distinct extensions are not necessarily the same thing as distinct bundles. In fact, elements of $H^{1}(\operatorname{Hom}(\mathcal{F}, \mathcal{G}))$ which differ only by an overall scale define isomorphic bundles.

Thus, isomorphism classes of non-split bundles are actually classified by $\operatorname{PExt}^{1}(\mathcal{F}, \mathcal{G})=\mathbf{P} H^{1}(\operatorname{Hom}(\mathcal{F}, \mathcal{G}))$.

How can we see this fact explicitly? Construct the bundles $\mathcal{F}, \mathcal{G}$, and $\mathcal{E}$ in terms of transition functions on overlaps of coordinate charts. The transition functions for $\mathcal{E}$ can be written in the form

$$
\left[\begin{array}{cc}
*_{\mathcal{F}} & A \\
0 & *_{\mathcal{G}}
\end{array}\right]
$$

where $*_{\mathcal{F}}$ and $*_{\mathcal{G}}$ are transition functions for $\mathcal{F}, \mathcal{G}$, respectively, and the $A$ 's on each overlap determine an element of $H^{1}(\operatorname{Hom}(\mathcal{F}, \mathcal{G}))$. Note that if $A=0$ on each coordinate overlap, then they define the zero element of $H^{1}(\operatorname{Hom}(\mathcal{F}, \mathcal{G}))$, and in particular the bundle $\mathcal{E}$ splits: $\mathcal{E}=\mathcal{F} \oplus \mathcal{G}$.

Suppose we multiply the element of $H^{1}(H o m(\mathcal{F}, \mathcal{G}))$ by a scalar $t$, then the effect is to multiply the $A$ in each transition function by $t$. We claim that the resulting bundle, call it $\mathcal{E}_{t}$, is isomorphic to the original bundle $\mathcal{E}$. To see this, simply change the local trivializations by multiplication by

$$
\left[\begin{array}{ll}
1 & 0 \\
0 & t
\end{array}\right]
$$

then the transition functions for $\mathcal{E}_{t}$ become those of $\mathcal{E}$ :

$$
\left[\begin{array}{ll}
1 & 0 \\
0 & t
\end{array}\right]\left[\begin{array}{cc}
*_{\mathcal{F}} & t A \\
0 & *_{\mathcal{G}}
\end{array}\right]\left[\begin{array}{cc}
1 & 0 \\
0 & t^{-1}
\end{array}\right]=\left[\begin{array}{cc}
*_{\mathcal{F}} & A \\
0 & *_{\mathcal{G}}
\end{array}\right]
$$

In other words, $\mathcal{E}_{t} \cong \mathcal{E}$ for $t \neq 0$, precisely as claimed.

Similar results hold when $\mathcal{F}$ and $\mathcal{G}$ are more general coherent sheaves, namely, elements of $\operatorname{Ext}^{1}(\mathcal{F}, \mathcal{G})$ that differ only by an overall scale define 
isomorphic sheaves. The argument is somewhat different than was used above in the special case of bundles; for completeness, we outline it here ${ }^{12}$.

First, consider an injective resolution of $\mathcal{G}$ :

$$
0 \longrightarrow \mathcal{G} \longrightarrow \mathcal{I}_{0} \stackrel{d_{0}}{\longrightarrow} \mathcal{I}_{1} \stackrel{d_{1}}{\longrightarrow} \cdots
$$

Now, calculate $\operatorname{Ext}^{1}(\mathcal{F}, \mathcal{G})$ as a right derived functor of $\operatorname{Hom}(\mathcal{F},-)$, namely, as the cohomology of the complex

$$
\operatorname{Hom}\left(\mathcal{F}, \mathcal{I}_{0}\right) \stackrel{d_{0}^{\prime}}{\longrightarrow} \operatorname{Hom}\left(\mathcal{F}, \mathcal{I}_{1}\right) \stackrel{d_{1}^{\prime}}{\longrightarrow} \operatorname{Hom}\left(\mathcal{F}, \mathcal{I}_{2}\right) \stackrel{d_{2}^{\prime}}{\longrightarrow} \cdots
$$

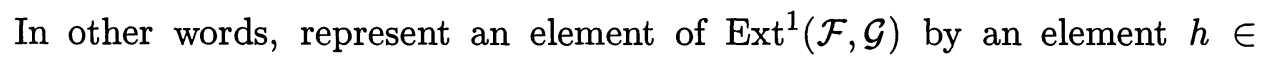
$\operatorname{Hom}\left(\mathcal{F}, \mathcal{I}_{1}\right)$ such that $d_{1}^{\prime}(h)=0$, modulo im $d_{0}^{\prime}$.

More concretely, given $h \in \operatorname{Hom}\left(\mathcal{F}, \mathcal{I}_{1}\right)$ such that $d_{1}^{\prime}(h)=0$, we have the following diagram

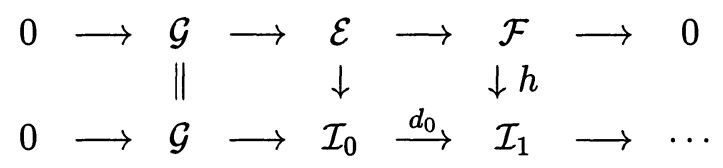

where the extension $\mathcal{E}$ is defined as follows. Local sections of $\mathcal{E}$ are given by pairs $(f, i)$, where $f$ is a local section of $\mathcal{F}$ and $i$ is a local section of $\mathcal{I}_{0}$ obeying the constraint $h(f)=d_{0}(i)$. The maps $\mathcal{E} \rightarrow \mathcal{F}$ and $\mathcal{E} \rightarrow \mathcal{I}_{0}$ are the obvious projections. Verification that the diagram above is commutative and of related details is left as an exercise for the reader.

We can now check that multiplying $h$ by a scalar $t$ yields an isomorphic sheaf. Define $h^{\prime}=t h$, and $\mathcal{E}_{t}$ the new extension. If $(f, i)$ is a local section of $\mathcal{E}$, then $(f / t, i)$ is a local section of $\mathcal{E}_{t}$, because $h(f)=h^{\prime}(f / t)$.

Thus for $t \neq 0, \mathcal{E}$ and $\mathcal{E}_{t}$ are isomorphic as sheaves, the isomorphism sending $(f, i)$ to $(f / t, i)$.

Finally, we should perhaps clarify why two sheaves that are isomorphic as sheaves need not be isomorphic as extensions. For two sheaves $\mathcal{E}_{1}$ and $\mathcal{E}_{2}$ to be isomorphic as extensions of $\mathcal{F}$ by $\mathcal{G}$ means that the following diagram must commute:

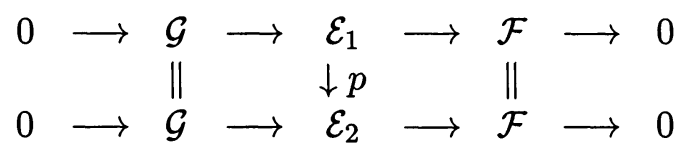

\footnotetext{
${ }^{12}$ We would like to thank T. Gomez for a useful discussion of this matter.
} 
where $p: \mathcal{E}_{1} \rightarrow \mathcal{E}_{2}$ is the isomorphism in question. It is easy to check that the isomorphism given above between $\mathcal{E}$ and $\mathcal{E}_{t}$ as sheaves does not yield a commutative diagram of the above form, thus they cannot be isomorphic as extensions.

\section{References}

[1] C. Okonek, M. Schneider, and H. Spindler, Vector Bundles on Complex Projective Spaces, Birkhauser, Boston, 1980.

[2] D. Huybrechts and M. Lehn, The Geometry of Moduli Spaces of Sheaves, Vieweg, Bonn, 1997.

[3] R. Friedman, Algebraic Surfaces and Holomorphic Vector Bundles, Springer-Verlag, New York, 1998.

[4] S. Kobayashi, Differential Geometry of Complex Vector Bundles, Princeton University Press, Princeton, 1987.

[5] S. K. Donaldson, "Anti Self Dual Yang-Mills Connections over Complex Algebraic Surfaces and Stable Vector Bundles," Proc. London Math. Soc. (3) 50 (1985) 1.

[6] K. Uhlenbeck and S. T. Yau, "On the Existence of Hermitian YangMills Connections in Stable Vector Bundles," Comm. Pure Appl. Math. 39 (1986) S257.

[7] E. Sharpe, "Notes on Heterotic Compactifications," Phys. Lett. B432 (1998) $32 \dot{6}$, hep-th/9710031.

[8] R. Blumenhagen, "(0,2) Target Duality, CICYs and Reflexive Sheaves," Nucl. Phys. B514 (1998) 688, hep-th/9710021.

[9] M. Duff, R. Minasian, and E. Witten, "Evidence for Heterotic / Heterotic Duality," Nucl. Phys. B465 (1996) 413, hep-th/9601036.

[10] A. Knutson, private communication.

[11] Z. Qin, "Chamber Structures of Algebraic Surfaces with Kodaira Dimension Zero and Moduli Spaces of Stable Rank Two Bundles," Math. Z. 207 (1991) 121.

[12] Z. Qin, "Birational Properties of Moduli Spaces of Stable Locally Free Rank-2 Sheaves on Algebraic Surfaces," Manuscripta Math. 72 (1991) 163. 
[13] Z. Qin, "Equivalence Classes of Polarizations and Moduli Spaces of Sheaves," J. Diff. Geom. 37 (1993) 397.

[14] Z. Qin, "Moduli of Stable Sheaves on Ruled Surfaces and their Picard Groups," J. Reine Angew. Math. 433 (1992) 201.

[15] R. Friedman and Z. Qin, "On Complex Surfaces Diffeomorphic to Rational Surfaces," Invent. Math. 120 (1995) 81.

[16] K. Matsuki and R. Wentworth, "Mumford-Thaddeus Principle on the Moduli Space of Vector Bundles on an Algebraic Surface," Int. J. Math. 8 (1997) 97.

[17] Y. Hu and W. Li, "Variation of the Gieseker and Uhlenbeck Compactifications," Int. J. Math. 6 (1995) 397.

[18] L. Göttsche, "Change of Polarization and Hodge Numbers of Moduli Spaces of Torsion-Free Sheaves on Surfaces," Math. Z. 223 (1996) 247.

[19] W. Li and Z. Qin, "Rank-3 Stable Bundles on Rational Ruled Surfaces," Math. Z. 222 (1996) 279.

[20] Z. Qin, private communication.

[21] I. Dolgachev and Y. Hu, "Variation of Geometric Invariant Theory Quotients," alg-geom/9402008.

[22] M. Thaddeus, "Geometric Invariant Theory and Flips," J. Amer. Math. Soc. 9 (1996) 691.

[23] R. Friedman and Z. Qin, "Flips of Moduli Spaces and Transition Formulas for Donaldson Polynomial Invariants of Rational Surfaces," Comm. Anal. Geom. 3 (1995) 11.

[24] G. Moore and E. Witten, "Integration over the $u$-Plane in Donaldson Theory," hep-th/9709193.

[25] A. Knutson and E. Sharpe, "Sheaves on Toric Varieties for Physics," hep-th/9711036.

[26] A. Knutson and E. Sharpe, "Equivariant Sheaves," hep-th/9804075.

[27] E. Witten, "Phases of $\mathrm{N}=2$ Theories in Two Dimensions," Nucl. Phys. B403 (1993) 159, hep-th/9301042.

[28] P. Aspinwall and D. Morrison, "String Theory on K3 Surfaces," hep-th/9404151, in Mirror Symmetry II, ed. B. Greene and S.-T. Yau. 
[29] P. Aspinwall, "K3 Surfaces and String Duality," hep-th/9611137.

[30] F. A. Bogomolov, "Holomorphic Tensors and Vector Bundles on Projective Varieties," Math. USSR Izv. 13 (1979) 499.

[31] M. Green, J. Schwarz, and E. Witten, Superstring Theory, vol. 2, Cambridge University Press, Cambridge, 1987.

[32] M. Dine, N. Seiberg, and E. Witten, "Fayet-Iliopoulos Terms in String Theory," Nucl. Phys. B289 (1987) 589.

[33] M. Douglas and G. Moore, "D-Branes, Quivers, and ALE Instantons," hep-th/9603167.

[34] T. Gomez, "Brill-Noether Theory on Singular Curves and Vector Bundles on K3 Surfaces," alg-geom/9710029, Princeton Ph.D. thesis.

[35] K. O'Grady, "The Weight-Two Hodge Structure of Moduli Spaces of Sheaves on a K3 Surface," J. Algebraic Geom. 6 (1997) 599, alg-geom/9510001.

[36] J. Erler, "Anomaly Cancellation in Six Dimensions," J. Math. Phys. 35 (4) (1994) 1819.

[37] J. Distler and B. Greene, "Aspects of $(2,0)$ String Compactifications," Nucl. Phys. B304 (1988) 1.

[38] R. Friedman, J. Morgan, and E. Witten, "Vector Bundles and F Theory," Comm. Math. Phys. 187 (1997) 679, hep-th/9701162.

[39] P. Aspinwall, "Aspects of the Hypermultiplet Moduli Space in String Duality," J. High Energy Phys. 04 (1998) 019, hep-th/9802194. 